九州大学学術情報リポジトリ

Kyushu University Institutional Repository

\title{
Tube domain and an orbit of a complex triangular group
}

Ishi, Hideyuki

Nomura, Takaak i

http://hdl. handle. net/2324/3334

出版情報 : Kyushu University Preprint Series in Mathematics, 2006. 九州大学大学院数理学研究院 バージョン：

権利関係: 


\title{
Tube domain and an orbit \\ of a complex triangular group
}

\author{
By Hideyuki IsHI and Takaaki NomurA
}

\begin{abstract}
Let $w$ be a complex symmetric matrix of order $r$, and $\Delta_{1}(w), \ldots, \Delta_{r}(w)$ the principal minors of $w$. If $w$ belongs to the Siegel right half space, then it is known that $\operatorname{Re}\left(\Delta_{k}(w) / \Delta_{k-1}(w)\right)>0$ for $k=1, \ldots, r$. In this paper we study this property in three directions. First we show that this holds for general symmetric right half spaces. Second we present a series of non-symmetric right half spaces with this property. We note that case-by-case verifications up to dimension 10 tell us that there is only one such irreducible non-symmetric tube domain. The proof of the property reduces to two lemmas. One is entirely generalized to non-symmetric cases as we prove in this paper. This is the third direction. As a byproduct of our study, we show that the basic relative invariants associated to a homogeneous regular open convex cone $\Omega$ studied earlier by the first author are characterized as the irreducible factors of the determinant of right multiplication operators in the complexification of the clan associated to $\Omega$.
\end{abstract}

\section{$\S 1$. Introduction.}

We begin this paper with an example in order to show what problem we consider. Let $V$ be the real vector space $\operatorname{Sym}(r, \mathbb{R})$ of $r \times r$ real symmetric matrices. We denote by $\Omega$ the cone of positive definite matrices in $V$. We have the corresponding tube domain $\Omega+i V$ in the complexification $W:=V_{\mathbb{C}}$. Obviously $W=\operatorname{Sym}(r, \mathbb{C})$, the space of $r \times r$ complex symmetric matrices. The complex general linear group $G L(r, \mathbb{C})$ acts on $W$ in the usual way: $G L(r, \mathbb{C}) \times W \ni(g, w) \mapsto g w^{\mathrm{t}} g$. Let $A_{\mathbb{C}}$ denote the subgroup of $G L(r, \mathbb{C})$ consisting of diagonal matrices, and $N_{\mathbb{C}}$ the subgroup of strictly lower triangular matrices. The complex triangular group mentioned in the title is $T_{\mathbb{C}}:=N_{\mathbb{C}} A_{\mathbb{C}}$. Let $\Delta_{1}(w), \ldots, \Delta_{r}(w)$ be the principal minors of the matrix 
$w \in W$. Thus if $w=\left(w_{i j}\right) \in W$, then

$$
\Delta_{k}(w)=\operatorname{det}\left(\begin{array}{ccc}
w_{11} & \cdots & w_{1 k} \\
\vdots & & \vdots \\
w_{k 1} & \cdots & w_{k k}
\end{array}\right) \quad(k=1,2, \ldots, r) .
$$

We know that $\Omega+i V$ is contained in the $T_{\mathbb{C} \text {-orbit }} T_{\mathbb{C}} \cdot I_{r}$ passing through the $r \times r$ identity matrix $I_{r}$. Therefore if $w \in \Omega+i V$, we have $\Delta_{k}(w) \neq 0$ for any $k=1,2, \ldots, r$. The following proposition is known.

Proposition 1.1. Let $w \in W$ and suppose $w \in \Omega+i V$. Then

$$
\operatorname{Re} \frac{\Delta_{k}(w)}{\Delta_{k-1}(w)}>0 \quad(k=1,2, \ldots, r),
$$

where we understand $\Delta_{0}(w) \equiv 1$.

Since Proposition 1.1 can be generalized to any symmetric cone $\Omega$ as we will show in Proposition 2.2 of this paper, it arises a question if the validity of Proposition 1.1 is characteristic of symmetric cones (or of symmetric tube domains). As a negative answer to this question, we will present in section 3 a series of non-symmetric homogeneous open convex cones for which, with a natural generalization of the principal minors $\Delta_{k}$, Proposition 1.1 remains true. Actually something more is done in this paper. To give details let us return to the example that we started with. First we would like to point out that the proof of Proposition 1.1 consists of two parts. The first one is the following lemma that can be shown by a direct computation.

Lemma 1.2. Let $w \in W$ and suppose $w \in T_{\mathbb{C}} \cdot I_{r}$. Then, writing $w$ as $w=n a^{t} n$ with $n \in N_{\mathbb{C}}$ and $a:=\operatorname{diag}\left[a_{1}, \ldots, a_{r}\right] \in A_{\mathbb{C}}$, one has

$$
a_{k}=\frac{\Delta_{k}(w)}{\Delta_{k-1}(w)} \quad(k=1, \ldots, r) .
$$

In section 2, we will present a proof of this Lemma in the general case where $W$ is the complexification of a Euclidean Jordan algebra. The idea of the proof does not differ much from the current example. The second one is:

Lemma 1.3. Suppose $n a^{t} n \in \Omega+i V$ for $n \in N_{\mathbb{C}}$ and $a=\operatorname{diag}\left[a_{1}, \ldots, a_{r}\right] \in A_{\mathbb{C}}$. Then

$$
\operatorname{Re} a_{1}>0, \ldots, \operatorname{Re} a_{r}>0
$$

Though Lemma 1.3 is further generalized to the case of homogeneous convex cones in section 4 , we think that it is worth giving a proof of Lemma 1.3 within symmetric cones by using the Jordan algebra language, and we do it in section 2. Combination 
of Lemmas 1.2 and 1.3 immediately yields Proposition 1.1 for symmetric cones. Also for the non-symmetric cones presented in section 3, we do have both of the lemmas, so that Proposition 1.1 holds.

In section 4, we generalize Lemma 1.3 as is already said. Thus it turns out that the validity of Proposition 1.1 depends solely on the validity of Lemma 1.2 for general homogeneous convex cones. Experiments tell us that the failure of Lemma 1.2 is a frequent occurrence for non-symmetric cases, and our examples in section 3 are rather exceptional. In fact we have verified that there is only one cone (i.e., our example in dimension 8) that has the property of Lemma 1.2 among the irreducible non-symmetric homogeneous convex cones for dimension up to 10 (see [4] for the classification).

In section 5, we present some applications of our study. The first one is the fact that the basic relative invariants associated to a homogeneous cone treated in [2] are characterized as the irreducible factors of the determinant of the right multiplication operators $R(w)$ in the complexification of the corresponding clan. The second one is to give another proof of the description of the cone given earlier by Rothaus [8] and $\mathrm{Xu}[10]$.

\section{$\S 2$. The case of symmetric cones.}

Our reference is the book Faraut-Korányi [1]. Let $V$ be a simple Euclidean Jordan algebra of rank $r$ with unit element $e$. For $x \in V$, we denote by $M(x)$ the multiplication operator ${ }^{1}$ by $x$. Thus $M(x) y=x y$ for $y \in V$. We take the trace inner product $\langle x \mid y\rangle:=\operatorname{tr}(x y)$ in $V$ as in [1], where tr denotes the trace function on the Jordan algebra $V$. Let $c_{1}, \ldots, c_{r}$ be a Jordan frame, so that $c_{1}+\cdots+c_{r}=e$. For $k=1, \ldots, r$, the Peirce 1 -space for the idempotent $c_{1}+\cdots+c_{k}$ will be denoted by $V^{(k)}$. Evidently we have $V^{(1)} \subset \cdots \subset V^{(r)}=V$. Let $P_{k}$ be the orthogonal projector $V \rightarrow V^{(k)}$. The space $V^{(k)}$ itself being a Euclidean Jordan algebra, we denote by $\operatorname{det}^{(k)}$ the determinant function of $V^{(k)}$. Let $\Delta_{k}(x):=\operatorname{det}^{(k)}\left(P_{k} x\right)$ be the $k$-th Jordan minor of $x \in V$. The Jordan frame $c_{1}, \ldots, c_{r}$ yields an orthogonal decomposition $V=\bigoplus_{j \leq k} V_{j k}$, where $V_{j j}=\mathbb{R} c_{j}(j=1, \ldots, r)$, and

$$
V_{j k}:=\left\{x \in V ; M\left(e_{i}\right) x=\frac{1}{2}\left(\delta_{i j}+\delta_{i k}\right) x \quad(i=1, \ldots, r)\right\} \quad(1 \leq j<k \leq r) .
$$

\footnotetext{
${ }^{1}$ The notation in the book [1] is $L(x)$. Since we use this for the left multiplication operator in the clan, we have chosen a different symbol.
} 
Let $\Omega:=\operatorname{Int}\left\{x^{2} ; x \in V\right\}$, the interior of squares in $V$. Then $\Omega$ is a symmetric cone and every irreducible symmetric cone arises in this way. Let $G(\Omega)$ be the linear automorphism group of the cone $\Omega$. We know that $G(\Omega)$ is reductive. Let $\mathfrak{g}$ be the Lie algebra of $G(\Omega)$. Let $\mathfrak{k}$ be the derivation algebra $\operatorname{Der}(V)$ of the Jordan algebra $V$. Put $\mathfrak{p}:=\{M(x) ; x \in V\}$. Then $\mathfrak{g}=\mathfrak{k}+\mathfrak{p}$ is a Cartan decomposition of $\mathfrak{g}$ with the corresponding Cartan involution $\theta X=-{ }^{t} X$. Let $\mathcal{A}$ be the $\mathbb{R}$-linear span of $c_{1}, \ldots, c_{r}$. Thus $\mathcal{A}:=\mathbb{R} c_{1} \oplus \cdots \oplus \mathbb{R} c_{r}$. Consider $\mathfrak{a}:=\{M(a) ; a \in \mathcal{A}\}$. Then $\mathfrak{a}$ is an abelian subalgebra which is maximal in $\mathfrak{p}$. Let $\alpha_{1}, \ldots, \alpha_{r}$ be the basis of $\mathfrak{a}^{*}$ dual to $M\left(c_{1}\right), \ldots, M\left(c_{r}\right)$. We know that the positive $\mathfrak{a}$-roots are $\frac{1}{2}\left(\alpha_{k}-\alpha_{j}\right)(k>j)$ and the corresponding root spaces $\mathfrak{g}_{\left(\alpha_{k}-\alpha_{j}\right) / 2}=: \mathfrak{n}_{k j}$ are described as

$$
\mathfrak{n}_{k j}:=\left\{z \square c_{j} ; z \in V_{j k}\right\},
$$

where $a \square b:=M(a b)+[M(a), M(b)]$. Summing up all of the $\mathfrak{n}_{k j}$ as $\mathfrak{n}:=\sum_{j<k} \mathfrak{n}_{k j}$, we have an Iwasawa decomposition $\mathfrak{g}=\mathfrak{k}+\mathfrak{a}+\mathfrak{n}$. Let $A:=\exp \mathfrak{a}$ and $N:=\exp \mathfrak{n}$, the subgroups of $G(\Omega)$ corresponding to $\mathfrak{a}$ and $\mathfrak{n}$ respectively.

Now let $W:=V_{\mathbb{C}}$, the complexification of $V$. The inner product $\langle\cdot \mid \cdot\rangle$ of $V$ is extended to a complex bilinear form on $W$, and we denote it by the same symbol. We have $W=\bigoplus_{j \leq k} W_{j k}$ with $W_{j k}:=\left(V_{j k}\right)_{\mathbb{C}}$. The complexifications of $A$ and $N$ are denoted by $A_{\mathbb{C}}$ and $N_{\mathbb{C}}$, respectively. Note that $A_{\mathbb{C}}$ and $N_{\mathbb{C}}$ are subgroups of $G L(W)$, and we shall put $T_{\mathbb{C}}:=N_{\mathbb{C}} A_{\mathbb{C}}$. From now on, the Jordan minors $\Delta_{k}(w)$ $(k=1, \ldots, r)$ are naturally continued to holomorphic polynomial functions on $W$.

For any idempotent $c \in W$ and an element $z \in W\left(c ; \frac{1}{2}\right)$, the Peirce $\frac{1}{2}$-space for $c$, we define complex Frobenius operators $\tau_{c}(z)$ on $W$ by the formula

$$
\tau_{c}(z):=\exp (2 z \square c) .
$$

These are unipotent operators (see [1, Lemma VI.3.1]).

Lemma 2.1. Let $w \in W$ and suppose that $w$ satisfies $\Delta_{k}(w) \neq 0$ for $k=1,2, \ldots, r$. Then one can find uniquely $z^{(j)} \in \bigoplus_{k=j+1}^{r} W_{j k}(1 \leq j \leq r-1)$ and $a_{1} \in \mathbb{C}^{\times}, \ldots, a_{r} \in$ $\mathbb{C}^{\times}$so that

$$
w=\tau_{c_{1}}\left(z^{(1)}\right) \tau_{c_{2}}\left(z^{(2)}\right) \ldots \tau_{c_{r-1}}\left(z^{(r-1)}\right)\left(a_{1} c_{1}+\cdots+a_{r} c_{r}\right) .
$$

Here note that $z^{(j)} \square c_{j} \in \sum_{k=j+1}^{r}\left(\mathfrak{n}_{k j}\right)_{\mathbb{C}}$.

Proof. Though the proof by induction on $r$ is totally parallel to that of $[1$, Theorem VI.3.5], we write it down for completeness. We have nothing to do for $r=1$, so supposing $r>1$, we assume the truth of the lemma for $r-1$. Let us decompose 
$w$ as $w=w_{1}+w_{1 / 2}+w_{0}$, where the $w_{j}$ is in the Peirce $j$-space $W\left(c_{1} ; j\right)$ for $c_{1}$ $(j=0,1 / 2,1)$. Then $w_{1}=\lambda c_{1}$ with $\lambda=\left\langle w \mid c_{1}\right\rangle=\Delta_{1}(w) \neq 0$. As in [1, Lemma VI.3.1], we have, for $z^{(1)} \in W\left(c_{1} ; \frac{1}{2}\right)$

$$
\tau_{c_{1}}\left(-z^{(1)}\right) w=w_{1}-2 M\left(z^{(1)}\right) w_{1}+w_{1 / 2}+w_{0}^{\prime}=\lambda c_{1}-\lambda z^{(1)}+w_{1 / 2}+w_{0}^{\prime}
$$

with some $w_{0}^{\prime} \in W\left(c_{1} ; 0\right)$. Therefore, there is unique $z^{(1)}:=\lambda^{-1} w_{1 / 2}$ such that $\tau_{c_{1}}\left(-z^{(1)}\right) w=\lambda c_{1}+w_{0}^{\prime}$. Since it holds that, for $k=2, \ldots, r$,

$$
\Delta_{k}(w)=\Delta_{k}\left(\lambda c_{1}+w_{0}^{\prime}\right)=\lambda \Delta_{k-1}^{\prime}\left(w_{0}^{\prime}\right),
$$

where $\Delta_{j}^{\prime}(j=1, \ldots, r-1)$ are the Jordan principal minor functions on $W\left(c_{1} ; 0\right)$, we see that $\Delta_{j}^{\prime}\left(w_{0}^{\prime}\right) \neq 0$ for any $j=1, \ldots, r-1$. Hence induction hypothesis yields that there are unique $z^{(j)}$ and $a_{j} \neq 0(j=2, \ldots, r)$ such that

$$
w_{0}^{\prime}=\tau_{c_{2}}\left(z^{(2)}\right) \cdots \tau_{c_{r-1}}\left(z^{(r-1)}\right)\left(a_{2} c_{2}+\cdots+a_{r} c_{r}\right) .
$$

Since $\left(z^{(j)} \square c_{j}\right) c_{1}=0$ for $j \geq 2$, we get

$$
\begin{aligned}
w & =\tau_{c_{1}}\left(z^{(1)}\right)\left(\lambda c_{1}+w_{0}^{\prime}\right) \\
& =\tau_{c_{1}}\left(z^{(1)}\right) \tau_{c_{2}}\left(z^{(2)}\right) \cdots \tau_{c_{r-1}}\left(z^{(r-1)}\right)\left(\lambda c_{1}+a_{2} c_{2}+\cdots+a_{r} c_{r}\right) .
\end{aligned}
$$

Putting $a_{1}:=\lambda$, we obtain the lemma.

Lemma 2.1 immediately implies that $w \in W$ satisfies $\Delta_{k}(w) \neq 0$ for any $k=$ $1, \ldots, r$ if and only if $w \in N_{\mathbb{C}} \cdot \mathcal{A}_{\mathbb{C}}^{\times}$, where

$$
\mathcal{A}_{\mathbb{C}}^{\times}:=\left\{a=\sum_{j=1}^{r} a_{j} c_{j} ; a_{j} \in \mathbb{C}^{\times}\right\} .
$$

Proposition 2.2. Let $w \in W$ and suppose $\Delta_{k}(w) \neq 0$ for any $k=1, \ldots, r$. Writing $w=n \cdot\left(a_{1} c_{1}+\cdots+a_{r} c_{r}\right)$ according to the above, one has

$$
a_{k}=\frac{\Delta_{k}(w)}{\Delta_{k-1}(w)} \quad(k=1, \ldots, r) .
$$

Proof. We put

$$
a=a_{1} c_{1}+\cdots+a_{r} c_{r}, \quad a^{(k)}=a_{1} c_{1}+\cdots+a_{k} c_{k}
$$

for simplicity. Lemma 2.1 tells us that we have

$$
n=\tau_{c_{1}}\left(z^{(1)}\right) \tau_{c_{2}}\left(z^{(2)}\right) \ldots \tau_{c_{r-1}}\left(z^{(r-1)}\right) .
$$

For each $k$, we extend $P_{k}$ to the projector $W \rightarrow W^{(k)}:=\left(V^{(k)}\right)_{\mathbb{C}}$. We shall show that, with $z_{k}^{(j)}:=P_{k}\left(z^{(j)}\right)$ for $j=1, \ldots, k-1$,

$$
P_{k}(n \cdot a)=\tau_{c_{1}}^{(k)}\left(z_{k}^{(1)}\right) \tau_{c_{2}}^{(k)}\left(z_{k}^{(2)}\right) \cdots \tau_{c_{k-1}}^{(k)}\left(z_{k}^{(k-1)}\right) a^{(k)}
$$


where the $\tau_{c_{j}}^{(k)}\left(z_{k}^{(j)}\right)(j=1, \cdots, k-1)$ are Frobenius operators on the Jordan algebra $W^{(k)}$. Then we get $\Delta_{k}(w)=a_{1} \cdots a_{k}$, and the proposition follows.

Now let $X \in\left(\mathfrak{n}_{t s}\right)_{\mathbb{C}}(s<t)$. If $i \leq j$, then we have (cf. [1, p. 109 (c)])

$$
X\left(W_{i j}\right) \subset \begin{cases}W_{t j} & (s=i<t<j), \\ W_{j t} & (s=i<j<t), \\ W_{i t} & (s=j) .\end{cases}
$$

For the remaining case $s \neq i, j$, we have $X\left(W_{i j}\right)=0$. Therefore, if $i>k$ or if $j>k$, then $X\left(W_{i j}\right) \subset\left(I-P_{k}\right) W$. Hence

$$
X\left(\left(I-P_{k}\right) W\right) \subset\left(I-P_{k}\right) W .
$$

This implies $\mathfrak{n}_{\mathbb{C}}\left(\left(I-P_{k}\right) W\right) \subset\left(I-P_{k}\right) W$, so that $N_{\mathbb{C}}\left(\left(I-P_{k}\right) W\right) \subset\left(I-P_{k}\right) W$. Next we look at $N_{\mathbb{C}}\left(P_{k} W\right)$. By (2.2) again, we see that if $s>k$ or if $t>k$, then we have $X\left(W_{i j}\right) \in\left(I-P_{k}\right) W$ for any $i \leq j$, so that $(\exp X) w \in w+\left(I-P_{k}\right) W$ for any $w \in W$. Consequently

$$
\tau_{c_{k}}\left(z^{(k)}\right) \cdots \tau_{c_{r-1}}\left(z^{(r-1)}\right) a \in a+\left(I-P_{k}\right) W .
$$

Now for $j=1, \ldots, k-1$, let us write $z^{(j)}$ as $z^{(j)}=z_{k}^{(j)}+\zeta_{j}$ with $\zeta_{j} \in \bigoplus_{m=k+1}^{r} W_{j m}$. If $j<m \leq k<m^{\prime}$, then, since $\left[\mathfrak{n}_{m j}, \mathfrak{n}_{m^{\prime} j}\right]=0$, the operators $\tau_{c_{j}}\left(z_{k}^{(j)}\right)$ and $\tau_{c_{j}}\left(\zeta_{j}\right)$ commute, and we have $\tau_{c_{j}}\left(z^{(j)}\right)=\tau_{c_{j}}\left(z_{k}^{(j)}\right) \tau_{c_{j}}\left(\zeta_{j}\right)$. Since

$$
\tau_{c_{j}}\left(\zeta_{j}\right) w \in w+\left(I-P_{k}\right) W \quad(\forall w \in W),
$$

and since $\tau_{c_{j}}\left(z_{k}^{(j)}\right)\left(P_{k} W\right) \subset P_{k} W$, we arrive at $(2.1)$.

Proposition 2.3. Let $n \in N_{\mathbb{C}}$ and $a_{1} \in \mathbb{C}^{\times}, \ldots, a_{r} \in \mathbb{C}^{\times}$. If $n \cdot\left(a_{1} c_{1}+\cdots+a_{r} c_{r}\right) \in$ $\Omega+i V$, then one has

$$
\operatorname{Re} a_{1}>0, \ldots, \operatorname{Re} a_{r}>0 \text {. }
$$

Proof. We prove the proposition by induction on $r$. For $r=1$, the proposition is trivially true. Thus we assume $r>1$, and suppose that the proposition is true for $r-1$. Put $w:=n \cdot\left(a_{1} c_{1}+\cdots+a_{r} c_{r}\right)$. The proof of Proposition 2.2 says

$$
P_{r-1} w=n^{\prime} \cdot\left(a_{1} c_{1}+\cdots+a_{r-1} c_{r-1}\right)
$$

with $n^{\prime}:=\tau_{c_{1}}^{(r-1)}\left(z_{r-1}^{(1)}\right) \tau_{c_{2}}^{(r-1)}\left(z_{r-1}^{(2)}\right) \cdots \tau_{c_{r-2}}^{(r-1)}\left(z_{r-1}^{(r-2)}\right)$. Clearly we have

$$
\operatorname{Re}\left(P_{r-1} w\right)=P_{r-1}(\operatorname{Re} w) \in \Omega_{r-1},
$$

where $\Omega_{r-1}$ is the symmetric cone in $P_{r-1}(V)=V^{(r-1)}$. Induction hypothesis tells us that $\operatorname{Re} a_{1}>0, \ldots, \operatorname{Re} a_{r-1}>0$. To get $\operatorname{Re} a_{r}>0$, we consider the Jordan algebra 
inverse $w^{-1}$ of $w$. We know that $w^{-1} \in \Omega+i V$ and that

$$
w^{-1}={ }^{\mathrm{t}} n^{-1} \cdot\left(a_{1}^{-1} c_{1}+\cdots+a_{r}^{-1} c_{r}\right),
$$

where ${ }^{t} n$ is taken with respect to the complex bilinear form $\langle\cdot \mid \cdot\rangle$. Let us denote by $Q_{r}$ the orthogonal projector $V \rightarrow \mathbb{R} c_{r}$, and we extend it to a complex linear operator on $W$, denoted by the same symbol $Q_{r}$. Evidently we have $Q_{r} w=\left\langle w \mid c_{r}\right\rangle c_{r}$, and thus we obtain

$$
\begin{aligned}
Q_{r}\left(w^{-1}\right) & =\left\langle{ }^{\mathrm{t}} n^{-1} \cdot\left(a_{1}^{-1} c_{1}+\cdots+a_{r}^{-1} c_{r}\right) \mid c_{r}\right\rangle c_{r} \\
& =\left\langle a_{1}^{-1} c_{1}+\ldots a_{r}^{-1} c_{r} \mid n^{-1} c_{r}\right\rangle c_{r} .
\end{aligned}
$$

Since (2.2) gives $X c_{r}=0$ for any $X \in \mathfrak{n}_{\mathbb{C}}$ (and thus $n^{-1} c_{r}=c_{r}$ in (2.3)), we get $Q_{r}\left(w^{-1}\right)=a_{r}^{-1} c_{r}$. Since we have

$$
\operatorname{Re} Q_{r}\left(w^{-1}\right)=Q_{r}\left(\operatorname{Re} w^{-1}\right) \in Q_{r}(\Omega)=\left\{\lambda c_{r} ; \lambda>0\right\}
$$

it holds that $\operatorname{Re} a_{r}^{-1}>0$, which obviously gives $\operatorname{Re} a_{r}>0$.

Note that $\Omega+i V$ is contained in $T_{\mathbb{C}} \cdot E=N_{\mathbb{C}} \cdot \mathcal{A}_{\mathbb{C}}^{\times}$(see [6, Proposition 2.8]). Then, combining Propositions 2.2 and 2.3, we get

Theorem 2.4. Let $w \in W$. If $w \in \Omega+i V$, then

$$
\operatorname{Re} \frac{\Delta_{k}(w)}{\Delta_{k-1}(w)}>0 \quad(k=1, \ldots, r) .
$$

Remark 2.5. It may be worth noting that the condition $\operatorname{Re} a_{j}>0$ for each $j$ does not imply that $n \cdot\left(a_{1} c_{1}+\cdots+a_{r} c_{r}\right) \in \Omega+i V$ for all $n \in N_{\mathbb{C}}$. In fact, consider $V=\operatorname{Sym}(2, \mathbb{R})$ and let $\Omega$ be the cone of positive definite matrices in $V$. We suppose that $a_{1}, a_{2} \in \mathbb{C}$ satisfy $\operatorname{Re} a_{1}>0$ and $\operatorname{Re} a_{2}>0$. Let $\xi \in \mathbb{R}$ and assume that $|\xi|>\left|a_{1}\right|^{-1} \sqrt{\left(\operatorname{Re} a_{1}\right)\left(\operatorname{Re} a_{2}\right)}$. Then a direct computation shows

$$
w:=\left(\begin{array}{cc}
1 & 0 \\
i \xi & 1
\end{array}\right)\left(\begin{array}{cc}
a_{1} & 0 \\
0 & a_{2}
\end{array}\right)\left(\begin{array}{cc}
1 & i \xi \\
0 & 1
\end{array}\right)=\left(\begin{array}{cc}
a_{1} & i a_{1} \xi \\
i a_{1} \xi & -a_{1} \xi^{2}+a_{2}
\end{array}\right) \notin \Omega+i V .
$$

Of course we have $\Delta_{1}(w)=a_{1}$ and $\Delta_{2}(w)=a_{1} a_{2}$. Therefore the converse of Theorem 2.4 is not true.

\section{$\S 3 . \quad$ Some non-symmetric cones.}

One might ask if Theorem 2.4 is characteristic of symmetric cones. The purpose of this section is to present a series of non-symmetric cones for which we have the same statement as Theorem 2.4 with natural $\Delta_{k}$ 's generalized to the case of nonsymmetric cones. 
In this section, $I_{n}$ will denote the identity matrix of the $n$-th order. We consider the following real vector space $V$, where $\mathbb{R}^{n}$ is considered as the space of real column vectors:

$$
V:=\left\{x=\left(\begin{array}{ccc}
x_{11} I_{n} & x_{21} I_{n} & \boldsymbol{y} \\
x_{21} I_{n} & x_{22} I_{n} & \boldsymbol{z} \\
{ }^{\mathrm{t}} \boldsymbol{y} & { }^{\mathrm{t}} \boldsymbol{z} & x_{33}
\end{array}\right) ; \begin{array}{l}
\boldsymbol{y} \in \mathbb{R}^{n}, \\
\boldsymbol{z} \in \mathbb{R}^{n},
\end{array} x_{i j} \in \mathbb{R}\right\} .
$$

Note that $V \subset \operatorname{Sym}(2 n+1, \mathbb{R})$. As an open convex cone we take

$$
\Omega:=\{x \in V ; x \text { is positive definite }\}
$$

If $n=1$, then $\Omega$ is nothing other than the symmetric cone of positive definite real symmetric matrices of order 3 . Thus we assume $n \geq 2$ throughout this section. Obviously we have $\operatorname{dim} \Omega=2 n+4$. To see that $\Omega$ is homogeneous, we take the following subgroups $A$ and $N$ of $G L(2 n+1, \mathbb{R})$ :

$$
\begin{aligned}
& A:=\left\{a=\left(\begin{array}{ccc}
a_{1} I_{n} & 0 & 0 \\
0 & a_{2} I_{n} & 0 \\
0 & 0 & a_{3}
\end{array}\right) ; a_{1}>0, a_{2}>0, a_{3}>0\right\}, \\
& N:=\left\{n=\left(\begin{array}{ccc}
I_{n} & 0 & 0 \\
\xi I_{n} & I_{n} & 0 \\
{ }^{t} \boldsymbol{n}_{1} & { }^{\mathrm{t}} \boldsymbol{n}_{2} & 1
\end{array}\right) ; \begin{array}{l}
\boldsymbol{n}_{1} \in \mathbb{R}^{n}, \\
\boldsymbol{n}_{2} \in \mathbb{R}^{n},
\end{array} \xi \in \mathbb{R}\right\} .
\end{aligned}
$$

Consider the semidirect product group $H:=N \rtimes A$. It is clear that $H$ acts on $\Omega$ by $H \times \Omega \ni(h, x) \mapsto \rho(h) x:=h x^{\mathrm{t}} h \in \Omega$. To see that this $H$-action is simply transitive, we solve the equation $x=n a^{t} n$ for a given $x \in \Omega$, where $a \in A$ and $n \in N$ with the expressions as in (3.2) are to be found. To describe the unique solution, we define polynomial functions $\Delta_{1}, \Delta_{2}, \Delta_{3}$ on $V$ by

$$
\left\{\begin{array}{l}
\Delta_{1}(x):=x_{11} \\
\Delta_{2}(x):=x_{11} x_{22}-x_{21}^{2}, \\
\Delta_{3}(x):=x_{11} x_{22} x_{33}+2 x_{21} \boldsymbol{y} \cdot \boldsymbol{z}-x_{33} x_{21}^{2}-x_{22}\|\boldsymbol{y}\|^{2}-x_{11}\|\boldsymbol{z}\|^{2},
\end{array}\right.
$$

where $\boldsymbol{y} \cdot \boldsymbol{z}$ denotes the canonical inner product in $\mathbb{R}^{n}$, and $\|\cdot\|$ is the corresponding Euclidean norm. Then, a straightforward computation yields

$$
\begin{array}{rlrlrl}
a_{1} & =\Delta_{1}(x), & a_{2} & =\frac{\Delta_{2}(x)}{\Delta_{1}(x)}, & & a_{3}=\frac{\Delta_{3}(x)}{\Delta_{2}(x)}, \\
\xi & =\frac{x_{21}}{\Delta_{1}(x)}, & \boldsymbol{n}_{1}=\frac{\boldsymbol{y}}{\Delta_{1}(x)}, & \boldsymbol{n}_{2} & =\frac{x_{11} \boldsymbol{z}-x_{21} \boldsymbol{y}}{\Delta_{2}(x)} .
\end{array}
$$

These formula together with the discussion of [2, Theorem 2.2] shows that $\Delta_{1}(x)$, $\Delta_{2}(x)$ and $\Delta_{3}(x)$ are the basic relative invariants associated to the cone $\Omega$. 
If $\delta_{k}(x)(k=1, \ldots, 2 n+1)$ stands for the $k$-th principal minor of the matrix $x \in V$ of order $2 n+1$ with the expression as in (3.1), then we have

$$
\delta_{k}(x)= \begin{cases}\Delta_{1}(x)^{k} & (1 \leq k \leq n), \\ \Delta_{1}(x)^{2 n-k} \Delta_{2}(x)^{k-n} & (n+1 \leq k \leq 2 n), \\ \Delta_{2}(x)^{n-1} \Delta_{3}(x) & (k=2 n+1) .\end{cases}
$$

Therefore it holds that for $x \in V$

$$
x \in \Omega \Longleftrightarrow \Delta_{k}(x)>0 \quad(k=1,2,3) .
$$

In what follows, we extend these $\Delta_{k}$ to holomorphic polynomial functions on $W:=$ $V_{\mathbb{C}}$. In order to have explicit expressions, we extend the inner product $\boldsymbol{y} \cdot \boldsymbol{z}$ to a complex bilinear form on $\mathbb{C}^{n}$. We set $\nu(\boldsymbol{y})=\boldsymbol{y} \cdot \boldsymbol{y}$. With this notation we have

$$
\left\{\begin{array}{l}
\Delta_{1}(x)=x_{11} \\
\Delta_{2}(x)=x_{11} x_{22}-x_{21}^{2} \\
\Delta_{3}(x)=x_{11} x_{22} x_{33}+2 x_{21} \boldsymbol{y} \cdot \boldsymbol{z}-x_{33} x_{21}^{2}-x_{22} \nu(\boldsymbol{y})-x_{11} \nu(\boldsymbol{z}) .
\end{array}\right.
$$

We now describe the dual cone of $\Omega$. Consider the vector space $V^{\prime}$ given as

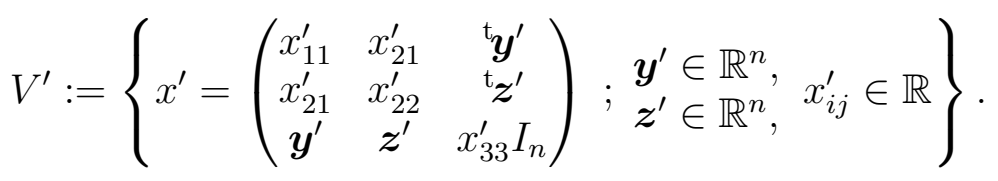

We note $V^{\prime} \subset \operatorname{Sym}(n+2, \mathbb{R})$ this time. We identify $V^{\prime}$ with the dual vector space of $V$ through

$$
\left\langle x, x^{\prime}\right\rangle=\sum_{j=1}^{3} x_{j j} x_{j j}^{\prime}+2 \boldsymbol{y} \cdot \boldsymbol{y}^{\prime}+2 \boldsymbol{z} \cdot \boldsymbol{z}^{\prime}+2 x_{21} x_{21}^{\prime},
$$

where $x$ and $x^{\prime}$ are as in (3.1) and (3.4) respectively. Consider the convex cone $\Omega^{\prime}$ of positive define ones in $V^{\prime}$ :

$$
\Omega^{\prime}:=\left\{x^{\prime} \in V^{\prime} ; x^{\prime} \text { is positive definite }\right\} .
$$

To see that $\Omega^{\prime}$ is homogeneous, we introduce the following subgroups $A^{\prime}, N^{\prime}$ of $G L(n+2, \mathbb{R})$ :

$$
\begin{aligned}
& A^{\prime}:=\left\{a^{\prime}=\left(\begin{array}{ccc}
a_{1} & 0 & 0 \\
0 & a_{2} & 0 \\
0 & 0 & a_{3} I_{n}
\end{array}\right) ; a_{1}>0, a_{2}>0, a_{3}>0\right\}, \\
& N^{\prime}:=\left\{n^{\prime}=\left(\begin{array}{ccc}
1 & \xi & { }^{\mathrm{t}} \boldsymbol{n}_{1} \\
0 & 1 & { }^{\mathrm{t}} \boldsymbol{n}_{2} \\
0 & 0 & I_{n}
\end{array}\right) ; \begin{array}{l}
\boldsymbol{n}_{1} \in \mathbb{R}^{2}, \\
\boldsymbol{n}_{2} \in \mathbb{R}^{2},
\end{array} \xi \in \mathbb{R}\right\} .
\end{aligned}
$$


Consider the semidirect product $H^{\prime}:=N^{\prime} \rtimes A^{\prime}$. Evidently $H^{\prime}$ acts on $\Omega^{\prime}$ by the rule $H^{\prime} \times \Omega^{\prime} \ni\left(h^{\prime}, x^{\prime}\right) \mapsto \rho^{\prime}\left(h^{\prime}\right) x^{\prime}:=h^{\prime} x^{\prime t} h^{\prime} \in \Omega^{\prime}$. This action is simply transitive. In fact, defining polynomial functions $\Delta_{1}^{\prime}, \Delta_{2}^{\prime}, \Delta_{3}^{\prime}$ by

$$
\left\{\begin{array}{l}
\Delta_{1}^{\prime}\left(x^{\prime}\right):=x_{33}^{\prime}, \\
\Delta_{2}^{\prime}\left(x^{\prime}\right):=x_{22}^{\prime} x_{33}^{\prime}-\left\|\boldsymbol{z}^{\prime}\right\|^{2}, \\
\Delta_{3}^{\prime}\left(x^{\prime}\right):=\left(x_{11}^{\prime} x_{33}^{\prime}-\left\|\boldsymbol{y}^{\prime}\right\|^{2}\right)\left(x_{22}^{\prime} x_{33}^{\prime}-\left\|\boldsymbol{z}^{\prime}\right\|^{2}\right)-\left(x_{21}^{\prime} x_{33}^{\prime}-\boldsymbol{y}^{\prime} \cdot \boldsymbol{z}^{\prime}\right)^{2},
\end{array}\right.
$$

we see by a straightforward computation that the unique solutions $n^{\prime} \in N^{\prime}$ and $a^{\prime} \in A^{\prime}$ (expressed as in (3.6)) of $x^{\prime}=n^{\prime} a^{\prime t} n^{\prime}$ for a given $x^{\prime} \in \Omega^{\prime}$ are written down explicitly as follows:

$$
\begin{aligned}
a_{1}=\frac{\Delta_{3}^{\prime}\left(x^{\prime}\right)}{\Delta_{1}^{\prime}\left(x^{\prime}\right) \Delta_{2}^{\prime}\left(x^{\prime}\right)}, & a_{2}=\frac{\Delta_{2}^{\prime}\left(x^{\prime}\right)}{\Delta_{1}^{\prime}\left(x^{\prime}\right)}, & a_{3}=\Delta_{1}^{\prime}\left(x^{\prime}\right), \\
\xi=\frac{x_{21}^{\prime} x_{33}^{\prime}-\boldsymbol{y}^{\prime} \cdot \boldsymbol{z}^{\prime}}{\Delta_{2}^{\prime}\left(x^{\prime}\right)}, & \boldsymbol{n}_{1}=\frac{\boldsymbol{y}^{\prime}}{\Delta_{1}^{\prime}\left(x^{\prime}\right)}, & \boldsymbol{n}_{2}=\frac{\boldsymbol{z}^{\prime}}{\Delta_{1}^{\prime}\left(x^{\prime}\right)} .
\end{aligned}
$$

Thus, just as in the case of $\Omega$, it holds that $\Delta_{1}^{\prime}\left(x^{\prime}\right), \Delta_{2}^{\prime}\left(x^{\prime}\right)$ and $\Delta_{3}^{\prime}\left(x^{\prime}\right)$ are the basic relative invariants associated to $\Omega^{\prime}$.

Let $\delta_{k}^{*}\left(x^{\prime}\right)(k=1, \ldots, n+2)$ denote the $k$-th principal minor of the matrix $x^{\prime} \in V^{\prime}$ of order $n+2$ written as in (3.4) that is taken from the $(n+2, n+2)$-entry to the $(n+3-k, n+3-k)$-entry. Then we have

$$
\delta_{k}^{*}\left(x^{\prime}\right)= \begin{cases}\Delta_{1}^{\prime}\left(x^{\prime}\right)^{k} & (1 \leq k \leq n) \\ \Delta_{1}^{\prime}(x)^{n-1} \Delta_{2}^{\prime}\left(x^{\prime}\right) & (k=n+1) \\ \Delta_{1}^{\prime}\left(x^{\prime}\right)^{n-2} \Delta_{3}^{\prime}\left(x^{\prime}\right) & (k=n+2)\end{cases}
$$

We just note that the expression for $\delta_{n+2}^{*}\left(x^{\prime}\right)=\operatorname{det} x^{\prime}$ follows from the identity

$$
\left(\begin{array}{cc}
A & B \\
C & \alpha I_{n}
\end{array}\right)\left(\begin{array}{cc}
I_{2} & 0 \\
-\alpha^{-1} C & I_{n}
\end{array}\right)=\left(\begin{array}{cc}
A-\alpha^{-1} B C & B \\
0 & \alpha I_{n}
\end{array}\right),
$$

where $A$ is $2 \times 2, B$ is $2 \times n, C$ is $n \times 2$ and $\alpha \neq 0$. Therefore we can conclude that for $x^{\prime} \in V^{\prime}$

$$
x^{\prime} \in \Omega^{\prime} \Longleftrightarrow \Delta_{k}^{\prime}\left(x^{\prime}\right)>0 \quad(k=1,2,3) .
$$

The functions $\Delta_{k}^{\prime}$ are extended to holomorphic polynomial functions on $W^{\prime}:=V_{\mathbb{C}}^{\prime}$ :

$$
\left\{\begin{aligned}
\Delta_{1}^{\prime}\left(x^{\prime}\right) & :=x_{33}^{\prime}, \\
\Delta_{2}^{\prime}\left(x^{\prime}\right) & :=x_{22}^{\prime} x_{33}^{\prime}-\nu\left(\boldsymbol{z}^{\prime}\right), \\
\Delta_{3}^{\prime}\left(x^{\prime}\right) & :=\left(x_{11}^{\prime} x_{33}^{\prime}-\nu\left(\boldsymbol{y}^{\prime}\right)\right)\left(x_{22}^{\prime} x_{33}^{\prime}-\nu\left(\boldsymbol{z}^{\prime}\right)\right)-\left(x_{21}^{\prime} x_{33}^{\prime}-\boldsymbol{y}^{\prime} \cdot \boldsymbol{z}^{\prime}\right)^{2} .
\end{aligned}\right.
$$

We now settle the problem of the dual cone of $\Omega$.

Proposition 3.1. $\Omega^{\prime}$ is the dual cone of $\Omega$ relative to the duality pairing (3.5):

$$
\Omega^{\prime}=\Omega^{*}:=\left\{x^{\prime} \in V^{\prime} ;\left\langle x, x^{\prime}\right\rangle>0 \quad \text { for any } x \in \bar{\Omega} \backslash\{0\}\right\} .
$$


Proof. We already know that $\Omega=\rho(H) I_{2 n+1}$ and $\Omega^{\prime}=\rho^{\prime}\left(H^{\prime}\right) I_{n+2}$. On the other hand, given $a_{k}>0(k=1,2,3), \boldsymbol{n}_{k} \in \mathbb{R}^{n}(k=1,2)$ and $\xi \in \mathbb{R}$, we take $a \in A$, $a^{\prime} \in A^{\prime}, n \in N$ and $n^{\prime} \in N^{\prime}$ as in (3.2) and (3.6), and put $h=n a$ and $h^{\prime}=a^{\prime} n^{\prime}$. Then a straightforward computation using

$$
\begin{aligned}
n & =\left(\begin{array}{ccc}
I_{n} & 0 & 0 \\
\xi I_{n} & I_{n} & 0 \\
0 & 0 & 1
\end{array}\right)\left(\begin{array}{ccc}
I_{n} & 0 & 0 \\
0 & I_{n} & 0 \\
0 & { }^{t} \boldsymbol{n}_{2} & 1
\end{array}\right)\left(\begin{array}{ccc}
I_{n} & 0 & 0 \\
0 & I_{n} & 0 \\
{ }^{t} \boldsymbol{n}_{1} & 0 & 1
\end{array}\right) \\
n^{\prime} & =\left(\begin{array}{ccc}
1 & 0 & { }^{\mathrm{t}} \boldsymbol{n}_{1} \\
0 & 1 & 0 \\
0 & 0 & I_{n}
\end{array}\right)\left(\begin{array}{ccc}
1 & 0 & 0 \\
0 & 1 & { }^{\mathrm{t}} \boldsymbol{n}_{2} \\
0 & 0 & I_{n}
\end{array}\right)\left(\begin{array}{ccc}
1 & \xi & 0 \\
0 & 1 & 0 \\
0 & 0 & I_{n}
\end{array}\right)
\end{aligned}
$$

gives us

$$
\left\langle\rho(h) x, x^{\prime}\right\rangle=\left\langle h x^{\mathrm{t}} h, x^{\prime}\right\rangle=\left\langle x, h^{\prime} x^{\prime \mathrm{t}} h^{\prime}\right\rangle=\left\langle x, \rho^{\prime}\left(h^{\prime}\right) x^{\prime}\right\rangle
$$

for $x \in V$ and $x^{\prime} \in V^{\prime}$. This implies that $\rho^{\prime}\left(H^{\prime}\right)$ is just the adjoint group of $\rho(H)$ relative to the duality pairing (3.5). Definition (3.5) tells us that $I_{n+2} \in \Omega^{*}$, and by general theory we know that $\Omega^{*}$ is an orbit of the adjoint group of $\rho(H)$. By these observations we conclude that $\Omega^{\prime}=\Omega^{*}$.

We note that $\Omega^{\prime}$ does not have the property of Theorem 2.4. In fact, consider

$$
w=\left(\begin{array}{ccc}
1+i & 0 & 0 \\
0 & 2+i & 0 \\
0 & 0 & (1+2 i) I_{n}
\end{array}\right) \in \Omega^{\prime}+i V^{\prime} .
$$

Then we have

$$
\operatorname{Re} \frac{\Delta_{3}^{\prime}(w)}{\Delta_{2}^{\prime}(w)}=\operatorname{Re}\{(1+i)(1+2 i)\}=-1<0 .
$$

Now we return to $\Omega$ and show that $\Omega$ has the property of Theorem 2.4. Let $A_{\mathbb{C}}$ and $N_{\mathbb{C}}$ be the complexifications of $A$ and $N$ respectively. We know by a general result (cf. [6, Proposition 2.8]) that $\Omega+i V \subset N_{\mathbb{C}} A_{\mathbb{C}} \cdot I_{2 n+1}$. Hence the functions $\Delta_{k}$ $(k=1,2,3)$ never vanish on $\Omega+i V$.

Theorem 3.2. Let $w \in V_{\mathbb{C}}$ and suppose $w \in \Omega+i V$. Then

$$
\operatorname{Re} \frac{\Delta_{k}(w)}{\Delta_{k-1}(w)}>0 \quad(k=1,2,3) .
$$

Proof. Given $w \in \Omega+i V$, we solve the equation $n a^{t} n=w$ for $n \in N_{\mathbb{C}}$ and $a \in A_{\mathbb{C}}$. Then, just as in (3.3) we get

$$
a_{k}=\frac{\Delta_{k}(w)}{\Delta_{k-1}(w)} \quad(k=1,2,3) .
$$


Since $\Omega$ is contained in the symmetric cone of positive definite real symmetric matrices of order $2 n+1$, we can apply Proposition 2.3 (or Lemma 1.3) to the present case. Hence we get $\operatorname{Re} a_{k}>0$, and the theorem follows.

\section{$\S 4$. A generalization.}

In this section we show that Proposition 2.3 is still true in the general case where $\Omega$ is a homogeneous regular open convex cone $\Omega$. Here, by regularity, we mean that $\Omega$ contains no entire line. Thanks to Vinberg [9], such cones are completely described by clans with unit element, and we begin this section with a brief description of clans.

Let $V$ be a finite-dimensional real vector space with a bilinear product $\triangle$ (the associative low is not required). The pair $(V, \triangle)$ is called a clan if the following three conditions are satisfied, where $L(x): y \mapsto x \triangle y$, the left multiplication by $x$ :

(C1) $[L(x), L(y)]=L(x \triangle y-y \triangle x)$,

(C2) There exists $s \in V^{*}$ such that $\langle x \triangle y, s\rangle$ defines an inner product in $V$,

(C3) Each $L(x)$ has only real eigenvalues.

Now, let $(V, \triangle)$ be a clan, and we will write $R(x)$ the right multiplication operator $y \mapsto y \triangle x$ by $x$. We suppose that $V$ has a unit element $E$. By (C1) above, we see that the set $\mathfrak{h}$ of left multiplication operators $L(x)(x \in V)$ forms a Lie subalgebra of $\mathfrak{g l}(V)$. By (C3), we see that $\mathfrak{h}$ is necessarily split solvable. We denote by $H$ the corresponding linear Lie group $\exp \mathfrak{h} \subset G L(V)$. Then, by [7, Thereom 4.15], the $H$-orbit $\Omega:=H \cdot E$ through $E$ is a regular open convex cone on which the group $H$ acts simply transitively. Vinberg's theory [9] tells us that every homogeneous regular open convex cone arises in this way.

Let $V=\bigoplus_{1 \leq j \leq k \leq r} V_{k j}$ be the normal decomposition of the clan $V$ with respect to a complete set of primitive idempotents $E_{1}, \ldots, E_{r}$ of $V$. This means that we have $E=E_{1}+\cdots+E_{r}$ and

$$
V_{k j}=\left\{x \in V ; L\left(E_{i}\right) x=\frac{1}{2}\left(\delta_{i k}+\delta_{i j}\right) x, R\left(E_{i}\right) x=\delta_{i j} x \quad \text { for } i=1, \ldots, r\right\} .
$$

Here we note $V_{k k}=\mathbb{R} E_{k}$ for any $k=1, \ldots, r$. We write $\mathcal{A}$ for the subspace $\mathbb{R} E_{1} \oplus$ $\cdots \oplus \mathbb{R} E_{r}$ of $V$, and $\mathcal{A}_{+}$for the set of elements $a_{1} E_{1}+\cdots+a_{r} E_{r} \in \mathcal{A}$ with $a_{i}>0$ for all $i=1, \ldots r$. Put $\mathfrak{n}:=[\mathfrak{h}, \mathfrak{h}] \subset \mathfrak{h}$ and $N:=\exp \mathfrak{n} \subset H$. Then we have $\Omega=N \cdot \mathcal{A}_{+}$.

Let $W$ be the complexification $V_{\mathbb{C}}$ of $V$. We extend the bilinear product $\triangle$ to $W$ by complex bilinearity. Accordingly, the left and the right multiplication operators are extended to $W$, and we use the same symbol $L(x)$ and $R(x)$ respectively for 
$x \in W$. Let $\mathfrak{n}_{\mathbb{C}} \subset \mathfrak{g l}(W)$ be the complexification of $\mathfrak{n}$, and $N_{\mathbb{C}} \subset G L(W)$ the corresponding complex linear Lie group $\exp \mathfrak{n}_{\mathbb{C}}$. We shall show the following:

Theorem 4.1. For $n \in N_{\mathbb{C}}$ and $a=a_{1} E_{1}+\cdots+a_{r} E_{r} \in \mathcal{A}_{\mathbb{C}}$, consider $z:=n \cdot a \in$ $N_{\mathbb{C}} \cdot \mathcal{A}_{\mathbb{C}} \subset W$. If $z$ belongs to the tube domain $\Omega+i V$, then

$$
\operatorname{Re} a_{1}>0, \ldots, \operatorname{Re} a_{r}>0 \text {. }
$$

To prove Theorem 4.1, we need more structural observations of $W$. Let $E_{1}^{*}, \ldots, E_{r}^{*}$ be the basis of $\mathcal{A}^{*}$ dual to $E_{1}, \ldots, E_{r}$. We extend these $E_{k}^{*}$ to $V^{*}$ by defining to be 0 on all of $V_{k j}(1 \leq j<k \leq r)$. For $s=\left(s_{1}, \ldots, s_{r}\right) \in \mathbb{R}^{r}$, we set $E_{s}^{*}=$ $s_{1} E_{1}^{*}+\cdots+s_{r} E_{r}^{*}$. We know that $\langle x \mid y\rangle:=\operatorname{tr} L(x \triangle y)$ defines an inner product in $V$. In fact we have $\langle x \mid y\rangle=\left\langle x \Delta y, E_{\boldsymbol{d}}^{*}\right\rangle$, where $\boldsymbol{d}=\left(d_{1}, \ldots, d_{r}\right)$ and

$$
d_{j}:=1+\frac{1}{2} \sum_{i<j} n_{j i}+\frac{1}{2} \sum_{k>j} n_{k j} \quad\left(n_{q p}:=\operatorname{dim} V_{q p} \text { for } q>p\right) .
$$

We note that the normal decomposition of $V$ is orthogonal with respect to $\langle\cdot \mid \cdot\rangle$.

Since $(\mathrm{C} 1)$ is rewritten as

$$
x \triangle(y \triangle z)-(x \triangle y) \triangle z=y \triangle(x \triangle z)-(y \triangle x) \triangle z \quad(x, y, z \in V),
$$

application of $E_{\boldsymbol{d}}^{*}$ to this formula yields

$$
\langle x \mid y \triangle z\rangle-\langle x \triangle y \mid z\rangle=\langle y \mid x \triangle z\rangle-\langle y \triangle x \mid z\rangle \quad(x, y, z \in V) .
$$

Let $W_{k j}(1 \leq j \leq k \leq r)$ be the complexification of $V_{k j}$. We record here the following multiplication relations for later references:

$$
W_{l k} \triangle W_{k j} \subset W_{l j},
$$

$$
\begin{gathered}
\text { if } k \neq i, j, \text { then } W_{l k} \triangle W_{i j}=0, \\
W_{l k} \triangle W_{m k} \subset W_{l m} \text { or } W_{m l} \text {, according to } l \geq m \text { or } m \geq l .
\end{gathered}
$$

For $k=1, \ldots, r$, we set $\mathcal{M}_{k}:=W_{k 1} \oplus \cdots \oplus W_{k, k-1} \oplus W_{k k}$. In view of (4.4), it holds that if $1 \leq k \leq l \leq r$, then

$$
\mathcal{M}_{k} \triangle \mathcal{M}_{l} \subset \mathcal{M}_{l}, \quad \mathcal{M}_{l} \triangle \mathcal{M}_{k} \subset \mathcal{M}_{l}
$$

Therefore, setting $\mathcal{I}_{k}:=\mathcal{M}_{k} \oplus \cdots \oplus \mathcal{M}_{r}(k=1, \ldots, r)$, we have an ideal sequence

$$
W=\mathcal{I}_{1} \supset \mathcal{I}_{2} \supset \cdots \supset \mathcal{I}_{r} \supset \mathcal{I}_{r+1}:=\{0\} .
$$

Put $m_{k}:=\operatorname{dim} \mathcal{M}_{k}(k=1, \ldots, r)$. Then we have $m_{k}=n_{k 1}+\cdots+n_{k, k-1}+1$. For $1 \leq j<k \leq r$, we fix an orthonormal basis $\left\{\boldsymbol{f}_{\alpha}^{k j}\right\}_{1 \leq \alpha \leq n_{k j}}$ of the subspace $V_{k j}$, and 
introduce an orthogonal basis $\left\{\boldsymbol{e}_{p}^{(k)}\right\}_{1 \leq p \leq m_{k}}$ of $\mathcal{M}_{k}$ by

$$
\boldsymbol{e}_{p}^{(k)}:=\left\{\begin{aligned}
\boldsymbol{f}_{\alpha}^{k j} & \left(p=\alpha+\sum_{i<j} n_{k i}, 1 \leq \alpha \leq n_{k j}\right), \\
\sqrt{2} d_{k}^{-1 / 2} E_{k} & \left(p=m_{k}\right) .
\end{aligned}\right.
$$

The natural isomorphism will be written as $\mathcal{M}_{k} \ni v \mapsto \dot{v}:=v+\mathcal{I}_{k+1} \in \mathcal{I}_{k} / \mathcal{I}_{k+1}$, and we write $\dot{\mathcal{M}}_{k}$ for the quotient space $\mathcal{I}_{k} / \mathcal{I}_{k+1}$. We denote by $R_{k}(x)\left(\right.$ resp. $\left.L_{k}(x)\right)$ the matrix of the linear operator on $\dot{\mathcal{M}}_{k}$ induced by $R(x)$ (resp. $L(x)$ ) with respect to the basis $\left\{\boldsymbol{e}_{p}^{(k)}\right\}_{1 \leq p \leq m_{k}}$ of $\dot{\mathcal{M}}_{k}$. For $a=\sum_{i=1}^{r} a_{i} E_{i} \in \mathcal{A}_{\mathbb{C}}$, we see by (4.1) that both $L_{k}(a)$ and $R_{k}(a)$ are diagonal matrices:

$$
\begin{aligned}
L_{k}(a) & =\left(\begin{array}{cccc}
\frac{1}{2}\left(a_{1}+a_{k}\right) I_{n_{k 1}} & & & \\
& \ddots & & \\
& & \frac{1}{2}\left(a_{k-1}+a_{k}\right) I_{n_{k, k-1}} & \\
& & & a_{k}
\end{array}\right), \\
R_{k}(a) & =\left(\begin{array}{llll}
a_{1} I_{n_{k 1}} & & & \\
& \ddots & & \\
& & a_{k-1} I_{n_{k, k-1}} & \\
& & & a_{k}
\end{array}\right)
\end{aligned}
$$

In the following the linear forms $E_{k}^{*}$ are extended to $W$ by complex linearity.

Lemma 4.2. For $x \in W$, one has

$$
R_{k}(x)=L_{k}(x)+{ }^{\mathrm{t}} L_{k}(x)-\left\langle x, E_{k}^{*}\right\rangle I_{m_{k}} \quad(k=1, \ldots, r) .
$$

In particular, $R_{k}(x)$ is a symmetric matrix.

Proof. Since the identity (4.7) is linear in $x$, it is enough to treat the following four cases separately:

(i) The case $x \in \mathcal{I}_{k+1}$.

In this case, both sides of (4.7) are zero matrices.

(ii) The case $x \in W_{k k}$.

In this case, (4.7) follows from (4.5) and (4.6).

(iii) The case $x=v \in \mathcal{M}_{k}^{0}:=\bigoplus_{j<k} W_{k j}$.

Since $\boldsymbol{e}_{m_{k}}^{(k)}=\sqrt{2} d_{k}^{-1 / 2} E_{k}$ by definition, (4.1) yields

$$
L(v) \boldsymbol{e}_{m_{k}}^{(k)}=\sqrt{2} d_{k}^{-1 / 2} R\left(E_{k}\right) v=0, \quad R(v) \boldsymbol{e}_{m_{k}}^{(k)}=\sqrt{2} d_{k}^{-1 / 2} L\left(E_{k}\right) v=\left(2 d_{k}\right)^{-1 / 2} v .
$$

On the other hand, we note that (4.4) tells us that $\mathcal{M}_{k}^{0} \triangle \mathcal{M}_{k}^{0} \subset W_{k k}=\mathbb{C} E_{k}$. Thus a simple observation shows that if $v_{1}, v_{2} \in \mathcal{M}_{k}^{0}$, then

$$
v_{1} \triangle v_{2}=d_{k}^{-1}\left\langle v_{1} \mid v_{2}\right\rangle E_{k}=\left(2 d_{k}\right)^{-1 / 2}\left\langle v_{1} \mid v_{2}\right\rangle \boldsymbol{e}_{m_{k}}^{(k)} .
$$


Now consider $v \in \mathcal{M}_{k}^{0}$. Since $\left\{\boldsymbol{e}_{p}^{(k)}\right\}_{1 \leq p \leq m_{k}-1}$ is an orthonormal basis of $\mathcal{M}_{k}^{0}$, we have $v=\sum_{p=1}^{m_{k-1}} v_{p} \boldsymbol{e}_{p}^{(k)}$ with $v_{p}=\left\langle v \mid \boldsymbol{e}_{p}^{(k)}\right\rangle$. Thus we see from (4.9) that

$$
L(v) \boldsymbol{e}_{p}^{(k)}=R(v) \boldsymbol{e}_{p}^{(k)}=\left(2 d_{k}\right)^{-1 / 2} v_{p} \boldsymbol{e}_{m_{k}}^{(k)}
$$

for $p=1, \ldots, m_{k}-1$. By (4.8) and (4.10) we get

$$
L_{k}(v)=\left(\begin{array}{cc}
O_{m_{k}-1} & 0 \\
\mathrm{t} \xi & 0
\end{array}\right), \quad R_{k}(v)=\left(\begin{array}{cc}
O_{m_{k}-1} & \xi \\
\mathrm{t} \xi & 0
\end{array}\right),
$$

where $\xi:=\left(1 / \sqrt{2 d_{k}}\right)^{\mathrm{t}}\left(v_{1}, \ldots, v_{m_{k}-1}\right) \in \mathbb{C}^{m_{k}-1}$. Since $\left\langle v, E_{k}^{*}\right\rangle=0$, we conclude that (4.7) holds in this case.

(iv) The case $x=y \in \bigoplus_{i \leq j<k} W_{j i}$.

We have $L(y) \boldsymbol{e}_{m_{k}}^{(k)}=R(y) \boldsymbol{e}_{m_{k}}^{(k)}=0$ by (4.1), and we see from (4.4) that the operators $L(y)$ and $R(y)$ preserve the space $\mathcal{M}_{k}^{0}$. Thus we obtain

$$
L_{k}(y)=\left(\begin{array}{cc}
L_{k}^{0}(y) & 0 \\
0 & 0
\end{array}\right), \quad R_{k}(y)=\left(\begin{array}{cc}
R_{k}^{0}(y) & 0 \\
0 & 0
\end{array}\right),
$$

where $L_{k}^{0}(y), R_{k}^{0}(y)$ are both matrices of order $m_{k}-1$. Now for $v_{1}, v_{2} \in \mathcal{M}_{k}^{0}$, we see from (4.3) that

$$
\left\langle v_{1} \mid L(y) v_{2}\right\rangle-\left\langle R(y) v_{1} \mid v_{2}\right\rangle=\left\langle y \mid v_{1} \triangle v_{2}\right\rangle-\left\langle L(y) v_{1} \mid v_{2}\right\rangle .
$$

Since $v_{1} \triangle v_{2} \in W_{k k}$, we have $\left\langle y \mid v_{1} \triangle v_{2}\right\rangle=0$. Hence (4.13) is rewritten as

$$
\left\langle R(y) v_{1} \mid v_{2}\right\rangle=\left\langle L(y) v_{1} \mid v_{2}\right\rangle+\left\langle v_{1} \mid L(y) v_{2}\right\rangle \quad\left(v_{1}, v_{2} \in \mathcal{M}_{k}^{0}\right) .
$$

This means that $R_{k}^{0}(y)=L_{k}^{0}(y)+{ }^{\mathrm{t}} L_{k}^{0}(y)$. Since $\left\langle y, E_{k}^{*}\right\rangle=0$, we arrive at (4.7) due to $(4.12)$.

Since (4.2) is rewritten as

$$
R(y \triangle z)=L(y) R(z)+R(z)(R(y)-L(y)),
$$

we see from Lemma 4.2 that

$$
R_{k}(y \triangle z)=L_{k}(y) R_{k}(z)+R_{k}(z)^{\mathrm{t}} L_{k}(y)-\left\langle y, E_{k}^{*}\right\rangle R_{k}(z) \quad(y, z \in W)
$$

for $k=1, \ldots, r$. Noting that the linear group $H_{\mathbb{C}}=\{\exp L(x) ; x \in W\}$ preserves the ideals $\mathcal{I}_{k}$ of $W$, we denote by $\Lambda_{k}(h)\left(h \in H_{\mathbb{C}}\right)$ the matrix of the linear operator on $\dot{\mathcal{M}}_{k}=\mathcal{I}_{k} / \mathcal{I}_{k+1}$ induced by the action of $h \in H_{\mathbb{C}}$ with respect to the basis $\left\{\dot{\boldsymbol{e}}_{p}^{(k)}\right\}_{1 \leq p \leq m_{k}}$ of $\dot{\mathcal{M}}_{k}$. Moreover, we see from (4.1) that

$$
x \triangle\left(E_{k}+\mathcal{I}_{k+1}\right) \subset\left\langle x, E_{k}^{*}\right\rangle E_{k}+\mathcal{I}_{k+1} \quad(x \in W),
$$

so that there exists a one-dimensional representation $\nu_{k}: H_{\mathbb{C}} \rightarrow \mathbb{C}^{\times}$such that

$$
h \cdot \dot{E}_{k}=\nu_{k}(h) \dot{E}_{k} \quad\left(h \in H_{\mathbb{C}}\right)
$$


with

$$
d \nu_{k}(L(x))=\left\langle x, E_{k}^{*}\right\rangle \quad(x \in W) .
$$

Then we have by (4.14)

$$
R_{k}(h \cdot z)=\nu_{k}(h)^{-1} \Lambda_{k}(h) R_{k}(z)^{\mathrm{t}} \Lambda_{k}(h) \quad\left(h \in H_{\mathbb{C}}, z \in W\right) .
$$

Lemma 4.3. If $x \in \Omega$, then $R_{k}(x)$ is a real positive definite symmetric matrix for $k=1, \ldots, r$.

Proof. We take $h \in H$ for which $x=h \cdot E$. Then we see from (4.17) that

$$
R_{k}(x)=\nu_{k}(h)^{-1} \Lambda_{k}(h)^{\mathrm{t}} \Lambda_{k}(h)
$$

because $R_{k}(E)$ is the identity matrix by (4.6). Since $\Lambda_{k}(h)$ is a real matrix, and since $\nu_{k}(h)>0$, Lemma 4.3 follows.

We are now in a position to prove Theorem 4.1.

Proof. Assume that $z=n \cdot a\left(n \in N_{\mathbb{C}}, a \in \mathcal{A}_{\mathbb{C}}\right)$ belongs to the tube domain $\Omega+i V$. By Lemma 4.3, we see that the real part of the complex symmetric matrix $R_{k}(z)$ is positive definite. Thus $R_{k}(z)$ is invertible and the real part of $R_{k}(z)^{-1}$ is also positive definite. On the other hand, we see from (4.17) that

$$
R_{k}(z)^{-1}=\nu_{k}(n)^{\mathrm{t}} \Lambda_{k}(n)^{-1} R_{k}(a)^{-1} \Lambda_{k}(n)^{-1} .
$$

Since $\nu_{k}$ is a one-dimensional representation, we have $\nu_{k}(n)=1$ for $n \in N_{\mathbb{C}}$. Hence (4.15) shows that the $m_{k}$-th column of $\Lambda_{k}(n)$ equals ${ }^{\mathrm{t}}(0, \ldots, 0,1)$ because $\boldsymbol{e}_{m_{k}}^{(k)}=$ $\sqrt{2} d_{k}^{-1 / 2} E_{k}$. Therefore (4.18) tells us that the $\left(m_{k}, m_{k}\right)$-component of $R_{k}(z)^{-1}$ is equal to the $\left(m_{k}, m_{k}\right)$-component of $R_{k}(a)^{-1}$, which is seen to be $a_{k}^{-1}$ by (4.6). Hence $\operatorname{Re} a_{k}^{-1}>0$, so that we obtain $\operatorname{Re} a_{k}>0$.

\section{$\S 5$. Application to relatively invariant polynomials.}

For the linear form $E_{s}^{*}$ introduced just before Theorem 4.1, We define a onedimensional representation $\chi_{\boldsymbol{s}}$ of $H$ by

$$
\chi_{\boldsymbol{s}}(\exp L(x)):=e^{\left\langle x, E_{s}^{*}\right\rangle} \quad(x \in V) .
$$

Recalling (4.16), we have $\nu_{k}=\chi_{s}$ for $s=(0, \ldots, \stackrel{(k)}{1}, \ldots, 0)$. Since $\operatorname{det} \Lambda_{k}(n)=1$ for $n \in N$, we see from (4.5) that

$$
\operatorname{det} \Lambda_{k}(h)=\nu_{k}(h)^{m_{k} / 2} \chi_{\sigma_{k} / 2}(h) \quad(h \in H),
$$


where $\sigma_{k}:=\left(n_{k 1}, n_{k 2}, \ldots, n_{k, k-1}, 1,0, \ldots, 0\right) \in \mathbb{Z}^{r}$. Then it follows from (4.17) that

$$
\operatorname{det} R_{k}(h \cdot x)=\chi_{\sigma_{k}}(h) \operatorname{det} R_{k}(x) \quad(h \in H, x \in V) .
$$

Let $\Delta_{k}(x)(k=1, \ldots, r)$ be the polynomials on $V$ determined by

$$
\Delta_{1}(x):=\operatorname{det} R_{1}(x), \quad \operatorname{det} R_{k}(x)=\Delta_{k}(x) \cdot \Delta_{1}(x)^{a_{k 1}} \Delta_{2}(x)^{a_{k 2}} \cdots \Delta_{k-1}(x)^{a_{k, k-1}}
$$

with the following two conditions for $k \geq 2$ :

(a) $a_{k 1}, a_{k 2}, \ldots, a_{k, k-1}$ are non-negative integers,

(b) $\Delta_{k}(x)$ is not divisible by any one of $\Delta_{1}(x), \ldots, \Delta_{k-1}(x)$.

Then we obtain the following theorem in a way completely parallel to the proof of [2, Theorem 2.2].

Theorem 5.1. The polynomials $\Delta_{1}(x), \ldots, \Delta_{r}(x)$ are the basic relative invariants on $V$ under the action of $H$.

From the definition of $R_{k}(x)$, it is evident that

$$
\operatorname{det} R(x)=\operatorname{det} R_{1}(x) \cdot \operatorname{det} R_{2}(x) \cdots \operatorname{det} R_{r}(x) \quad(x \in V) .
$$

Thus we can write

$$
\operatorname{det} R(x)=\Delta_{1}(x)^{a_{1}} \Delta_{2}(x)^{a_{2}} \cdots \Delta_{r}(x)^{a_{r}}
$$

with positive integers $a_{1}, \ldots, a_{r}$ thanks to (5.2). Therefore, the relative invariants $\Delta_{1}(x), \ldots, \Delta_{r}(x)$ are characterized as the irreducible factors of the polynomial $\operatorname{det} R(x)$. We note that $\operatorname{deg} \Delta_{k}(x)=\operatorname{deg} \Delta_{k-1}(x)$ is a possibility. Indeed this is already the case for the Vinberg cone and its dual cone (see [2]).

Remark 5.2. Consideration of the determinant of right multiplication operators for the study of relatively invariant polynomials appears also in [5, Corollary 2.20].

Proposition 5.3. One has

$$
\Omega=\left\{x \in V ; R_{k}(x) \text { is positive definite for } k=1, \ldots, r\right\} .
$$

Proof. Assume that the matrix $R_{k}(x)$ is positive definite for all $k=1, \ldots, r$. Then we have $\operatorname{det} R_{k}(x)>0$, so that $\Delta_{k}(x)>0$ owing to (5.2). Therefore $x$ belongs to $\Omega$ because

$$
\Omega=\left\{x \in V ; \Delta_{k}(x)>0(k=1, \ldots, r)\right\}
$$

by [2, Proposition 2.3]). Hence Proposition 5.3 follows from Lemma 4.3.

Remark 5.4. One can show that the description of $\Omega$ in Proposition 5.3 coincides with the ones given by Rothaus [8] and $\mathrm{Xu}[10]$ (see [3]). 


\section{References}

[1] J. Faraut and A. Korányi, Analysis on symmetric cones, Clarendon Press, Oxford, 1994.

[2] H. Ishi, Basic relative invariants associated to homogeneous cones and applications, J. Lie Theory, 11 (2001), 155-171.

[3] - On symplectic representations of normal $j$-algebras and their application to Xu's realizations of Siegel domains, to appear in Differential Geom. Appl.

[4] S. Kaneyuki and T. Tsuji, Classification of homogeneous bounded domains of lower dimension, Nagoya Math. J. 53 (1974), 1-46.

[5] T. Kimura, Introduction to prehomogeneous vector spaces, Translations of Math. Monogr., 215, American Mathematical Society, Providence, RI, 2003.

[6] T. Nomura, On Penney's Cayley transform of a homogeneous Siegel domain, J. Lie Theory, 11 (2001), 185-206.

[7] H. Rossi and M. Vergne, Representations of certain solvable Lie groups on Hilbert spaces of holomorphic functions and the application to the holomorphic discrete series of a semisimple Lie group, J. Funct. Anal., 13 (1973), $324-389$.

[8] O. S. Rothaus, The construction of homogeneous convex cones, Ann. of Math., 83 (1966), 358-376, Correction: ibid, 87 (1968), 399.

[9] E. B. Vinberg, The theory of convex homogeneous cones, Trans. Moscow Math. Soc., 12 (1963), 340-403.

[10] Y. C. Xu, Automorphism groups of homogeneous bounded domains, Acta Math. Sinica, 19 (1976), 169-191 (in Chinese). 